of such recordings. While recognising his reasons for focusing on these issues, I feel it important to point out that patients taperecording interviews can be a positive part of their therapy. Over the last seven and a half years as a consultant, I have had a number of patients who have taped sessions with me so as to allow the time to reflect upon the content of the sessions.

Attending out-patient sessions or indeed any other therapeutic interaction can be stressful so the ability to take in information can be impaired. For these patients, the ability to tape-record their interactions with me was valuable in that it enabled them to go over issues they could not clearly remember or to use the tapes to help them to write down questions which they could put at their next interview. While having a tape recorder going could influence the nature of the interaction, I found that it much less intrusive than patients trying to write down key issues by hand which made the interviews much more stilted.

I would agree with Dr Stephenson that taperecordings do not take the place of patients being able to read their own notes and do have an uncertain legal status, but I feel it important to recognise that if a patient wishes to have a tape recording, then this should be approached positively as it may have important therapeutic benefits.

Jan A. Davidson, North Mersey Community (NHS) Trust, Acute Directorate, Sefton General Hospital, Liverpool L15 2HE

\section{A model for an integrated psychotherapy service}

Sir: We were interested to read Drs Holmes \& Mitchison's article proposing a model for an integrated psychotherapy service (Psychiatric Bulletin, April 1994, 19, 209-213). In the inner city area of City and Hackney we are building a Department of Psychological Therapies which closely mirrors the model proposed. In our service the consultants, a psychoanalyst and a cognitive psychotherapist work with dynamic and behavioural specialist nurse therapists, a specialist nurse counsellor and a psychologist as a core team. As a young department we are learning to work together while maintaining our individual identities, but unlike Holmes \& Mitchison, do not see this as our main problem. Our major difficulty is of obtaining resources. The model we are aspiring to cannot be financed simply by psychotherapists altering their working practices; is a radical move outwards which, to be done properly, needs adequate financing. There are some similarities to the move to the community of general psychiatric services. It now seems to be well recognised that without adequate planning and resourcing it is sadly too easy to be in a position of providing a less caring and less effective service to our patients. We hope we can apply some of these painfully gained lessons to our own service.

We welcome Holmes \& Mitchison's suggestions as to ways to address these issues and would be very interested in hearing of other departments' experiences.

SIOBHAN MURPHY and STIRLING MORREY, Department of Psychological Therapies, City and Hackney Community Services NHS Trust, St Bartholomew's Hospital, William Harvey House, 61 Bartholomew Close, London EC1A $7 B E$

\section{Overseas training experience}

Sir: I support Ruth McCutcheon's comments (Psychiatric Bulletin, March 1995, 19, 161162) about the value of an exchange of trainees between the UK and other countries. She highlighted the teaching role of UK trainees in sub-specialities, and I would add that this should be a mutual exercise, involving an exchange of clinical and academic ideas.

Singapore, my country of origin, is an interesting example in examining how the sub-specialities are practised. As described by Robertson et al (1992), learning disability does not fall entirely within the remit of psychiatry; voluntary associations mainly provide for the learning disabled.

Drug rehabilitation centres (DRC) are run by the Prisons Department. Addictive behaviour specialists would be keen to argue that psychiatry should figure more prominently; an exchange programme would offer insight into the workings of the DRCs.

Forensic psychiatry provides another insightful exercise. The equivalent of a medium secure unit (360 beds!) operates in Singapore's only government psychiatric hospital (Singapore's population is 3 million). Meanwhile, there is a maximum secure psychiatric facility within the Hospital Wing of Changi Prison. Here, there is unique 